\title{
CISTITIS GLANDULAR FLORIDA DE TIPO INTESTINAL CON EXTRAVASACIÓN DE MUCINA: UNA LESIÓN QUE SIMULA UN TUMOR
}

\author{
R. GUARCH TROYAS, J. JIMÉNEZ CALVO*, B. REPARAZ ROMERO, \\ M.L. GÓMEZ DORRONSORO
}

Servicio de Anatomía Patológica. *Servicio de Urología. Hospital Virgen del Camino. Pamplona (Navarra).

Actas Urol Esp. 27 (4): 297-300, 2003

\section{RESUMEN}

"CISTITIS GLANDULAR FLORIDA DE TIPO INTESTINAL CON EXTRAVASACIÓN DE MUCINA: UNA LESIÓN QUE SIMULA UN TUMOR"

En la vejiga se pueden observar una gran variedad de lesiones o cambios metaplásicos epiteliales. La sustitución extensa del urotelio por epitelio similar a la mucosa intestinal se ha considerado como una lesión premaligna. Sin embargo, la historia natural de la metaplasia intestinal y su comportamiento a largo plazo es desconocida. Presentamos un caso de cistitis glandular de tipo intestinal vejiga en un paciente joven. En la cistoscopia se observó un tumor bien circunscrito de $4 \mathrm{~cm}$ localizado en trígono. Histológicamente la lesión mostraba numerosas glándulas revestidas por epitelio de tipo intestinal sin atipia citológica correspondiente a la variedad intestinal de la cistitis glandular. Destacaba en este caso el depósito de un material basófilo en el estroma correspondiente a mucina. Se podía ver acúmulos de este material rodeados y comprimidos por tejido conectivo, simulando quistes mucosos. Este caso demuestra que en el estudio cistoscópico la cistitis glandular puede simular un tumor cuando la lesión es extensa. Y que la extravasación de mucina puede causar un problema de diagnóstico diferencial con un tumor maligno, un hallazgo que ha sido raramente documentado previamente. Se discute además el significado biológico de ese tipo de lesión.

PALABRAS CLAVE: Vejiga. Cistitis glandular. Variante intestinal. Metaplasia intestinal. Extravasación de mucina. Lesión seudotumoral.

\section{ABSTRACT}

"FLORID CYSTITIS GLANDULARIS OF INTESTINAL TYPE WITH MUCIN EXTRAVASATION:

\section{A MIMIC OF TUMOUR"}

A wide spectrum of glandular epitheliales metaplastic changes may be seen in the bladder. Extensive replacement of the urotelium with an epithelium resembling intestinal mucosa is recognised as a premalignant condition. However, the natural history of intestinal metaplasia of the urinary bladder and longterm outcome are unknown. We report a case of glandular cystitis with intestinal metaplasia in a young patient. Cistoscopy revealed a tumour well circumscribed measuring $4 \mathrm{~cm}$ on the trigone. Microscopic examination showed numerous glands lined by intestinal type epithelium without atipia, conforming to the appearence of the intestinal variant of cystitis glandularis. This case had prominent foci of basophilic mucin in the stroma. Rounded aggregates of mucin were occasionally surrounded by compressed conective tissue cells, simulating mucinous cyst. This case illustrates the extent to wich cystitis glandularis may mimic a neoplasm on gross evaluation and the propensity of mucin extravasation to cause diagnostic difficulty, a finding documented only rarely previously, and we discuss its biologic significance.

KEY WORDS: Urinary bladder. Cystitis glandularis. Intestinal variant. Intestinal metaplasia. Tumor like lesion 
$\mathrm{L}$ as lesiones vesicales que por su expresión clínica se pueden confundir con tumores son frecuentes. Raramente la cistitis glandular puede sugerir un tumor. Esto ocurre en aquellos casos en que la lesión es florida, correspondiendo habitualmente a la variante intestinal de este tipo de lesión.

Dicha enfermedad, también llamada metaplasia intestinal (MI) de la vejiga corresponde a una proliferación anormal de glándulas de tipo colónico en ese órgano. En los casos que la lesión es extensa $^{1}$, se le considera una lesión potencialmente maligna. Su incidencia aproximada es del $0,1-0,9 \%^{2,3}$, se asocia frecuentemente a la extrofia vesical ${ }^{4}$ y a la lipomatosis pélvica ${ }^{5}$ y tiene una localización preferentemente en trígono. Presentamos un caso de este tipo de lesión en un paciente joven que clínicamente fue diagnosticado de un tumor vesical y en el que se realizó estudio anatomopatológico de la lesión con seguimiento durante dos años.

\section{CASO CLÍNICO}

Varón de 32 años sin antecedentes personales ni familiares de interés, que debutó con hematuria y síndrome miccional. En la cistoscopia, se observó una lesión tumoral sesil extensa de $4 \mathrm{~cm}$ en el trígono que se biopsió (Fig. 1). Histológicamente y a bajo aumento, la mayor parte de los fragmentos obtenidos mostraron un crecimiento polipoide de la mucosa, el revestimiento urotelial en superficie se hallaba sustituido por una proliferación de glándulas semejante al epitelio colónico (Fig. 2). Entremezclado con dichas glándulas se observó un material basófilo que incluso llegaba a conformar grandes lagos de moco en la lámina propia que recordaban a quistes mucosos delimitados por tejido conectivo, donde no se encontraban células epiteliales (Fig. 3). La proliferación glandular adyacente estaba constituida por células columnares productoras de moco que no mostraban pseudoestratificación ni atipia citológica (Fig. 4). La distribución de las glándulas era ordenada y no existía un patrón infiltrativo. Dichas glándulas se teñian con el PAS y el azul alcián. El diagnóstico de la lesión fue de cistitis glandular de tipo intestinal. Tres meses más tarde la lesión nuevamente había crecido, y se extirpó en su totalidad mediante resección transuretral (RTU). Histológicamente el mate-

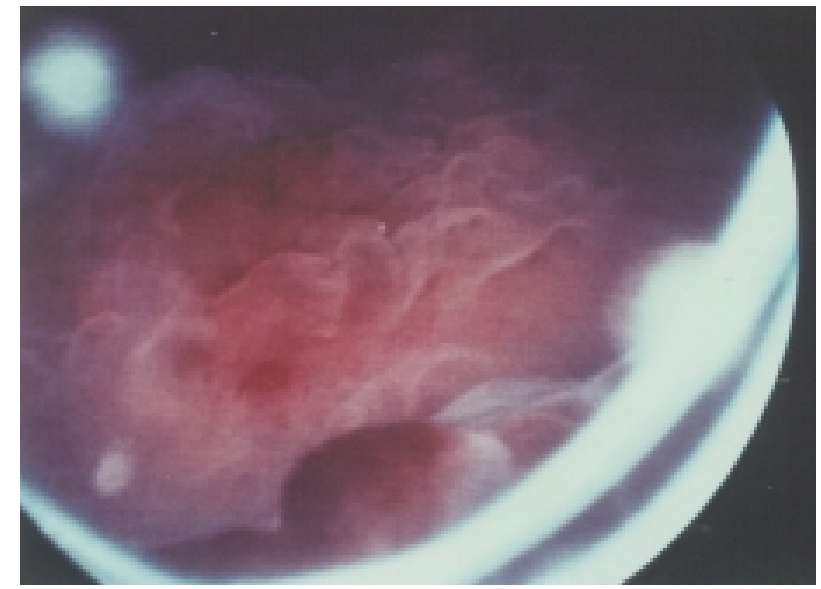

FIGURA 1. Imagen cistoscópica de tumoración sesil extensa en trigono.

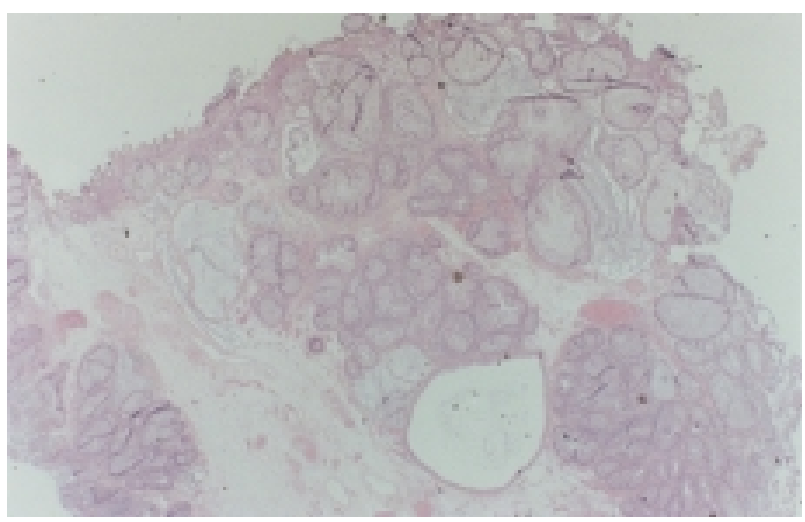

FIGURA 2. Imagen polipoide de la mucosa con sustitución del urotelio en superficie y en profundidad por glándulas de tipo colónico.

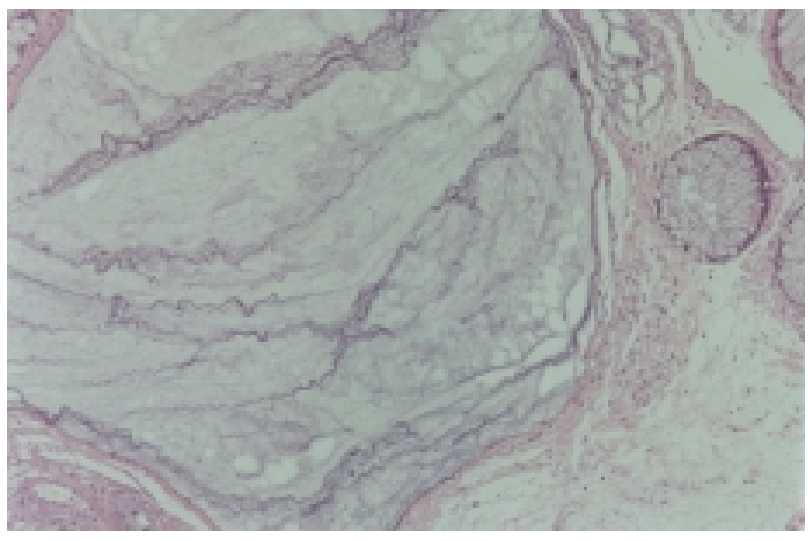

FIGURA 3. Grandes depósitos de mucina sin células epiteliales próximos a las glándulas.

rial mostró dos áreas morfológicamente diferentes, que se delimitaban bien. Por una parte se encontraban áreas superponibles a las anteriormente descritas y en otras áreas podía verse la 


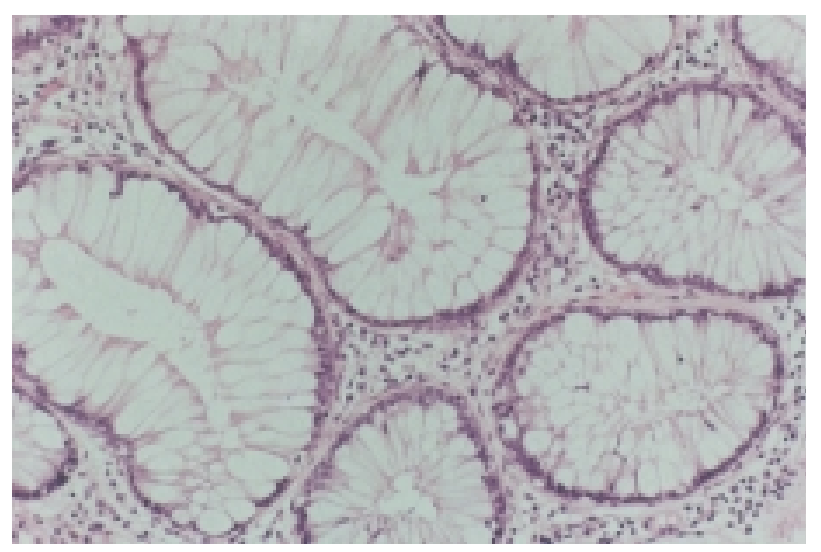

FIGURA 4. A mayor aumento, proliferación glandular constituida por células columnares productoras de moco sin seudoestratificación ni atipia citológica.

conservación en superficie del epitelio urotelial y en profundidad una proliferación glandular diferente, como corresponde a una cistitis glandular en su forma "típica", con glándulas constituidas por células columnares sin mucina rodeadas por varias capas de células uroteliales. El diagnóstico de esta segunda biopsia fue de cistitis glandular de tipo intestinal (50\%) y cistitis glandular típica (50\%). Un año más tarde se realizó reimplantación ureteral con biopsia vesical normal. 2 años más tarde del primer diagnóstico, la lesión de nuevo había recidivado encontrándose alteraciones de cistitis glandular de tipo intestinal superponibles a las observadas en la primera biopsia.

\section{DISCUSIÓN}

La metaplasia intestinal de la vejiga es una lesión rara; puede ser confundida con un tumor cuando la lesión es extensa y difusa. En esos es necesaria la realización de una biopsia para llegar al diagnóstico definitivo. Este tipo de lesión se caracteriza por glándulas con células columnares con abundante mucina intracitoplasmática, frecuentemente con células goblet, células de panneth, argentafines y argirófilas ${ }^{6}$, rasgos todos ellos superponibles a los observados en este caso.

A lo largo de los años no ha habido un acuerdo unánime acerca de la nomenclatura para esta lesión, se le ha denominado cistitis glandular de tipo intestinal, metaplasia intestinal, metaplasia glandular y tejido gastrointestinal aberrante.

En el caso descrito en este trabajo destaca la extravasación de mucina, dicho rasgo ha sido des- crito en 10 casos en la literatura ${ }^{7}$, y puede plantear un problema de diagnóstico diferencial con entidades como el adenocarcinoma mucinoso y la endocervicosis ${ }^{7}$. La ausencia de atipia citológica, y de mucina entremezclada con células epiteliales descartan un tumor maligno, y la ausencia de una respuesta fibroblástica e histiocitaria, junto al origen epitelial de tipo mülleriano descartan la segunda posibilidad.

Muchas teorías han sido propuestas para la etiología de esta lesión. Sin embargo únicamente dos han sido ampliamente aceptadas. La primera de ellas sugiere que el agente etiológico es la irritación del urotelio, por causa infecciosa o de otro tipo. Y cuyo mecanismo patogenético es que el urotelio irritado, prolifera en forma de nidos epiteliales (nidos de Von Brunn) produciéndose frecuentemente la formación de quistes en dichos nidos (cistitis quística) y metaplasia glandular en forma de glándulas productoras de $\operatorname{moco}^{6}$. Otra teoría sugiere que nidos de células intestinales de origen endodérmico persisten en la vejiga durante la embriogénesis después de que el recto se ha separado del tracto urogenital. En este caso no se encontró ningún factor causante que estuviera relacionado con este tipo de lesión.

La edad media de presentación de la metaplasia intestinal es en gente más joven que el carcinoma de vejiga ${ }^{1}$. La historia natural de la metaplasia intestinal y su comportamiento a largo plazo es desconocido. Muchos autores la han considerado como una lesión reactiva a una neoplasia y otros como una lesión premaligna por su asociación a tumores malignos ${ }^{1,2,8}$. Dichos casos han sido referidos mayoritariamente en la literatura como coexistencia entre metaplasia intestinal y tumor maligno, frecuentemente adenocarcinoma. Si la MI corresponde a una lesión extensa y difusa constituye un factor de alto riesgo para el desarrollo de adenocarcinoma ${ }^{1}$. La recurrencia de este tipo de lesión como ocurre en el caso que presentamos ha sido descrita en la literatura ${ }^{9}$; asi como la progresión de MI-displasia-adenocarcinoma ha sido descrita en algunos pacientes con biopsias secuenciales durante 5-15 años ${ }^{2,8,10-12}$.

Contrariamente, desde un punto de vista epidemiológico, el estudio de Corica y cols. ${ }^{4}$ recoge 53 casos de pacientes con extrofia vesical y MI, seguidos durante 12 años. Dichos autores no encon- 
traron ningún caso que evolucionara a tumor maligno, por lo que sugieren que la MI no es un factor de alto riesgo para el desarrollo de un adenocarcinoma. Un paso más y desde un punto de vista especulativo, los estudios por inmunohistoquímica del antígeno mabDAS ${ }^{13,14}$, demuestran que dicho antígeno se expresa en mucosa colónica normal y en tejido intestinal aberrante benigno y maligno. Estos resultados sugieren que el adenocarcinoma en vejiga procede de tejido metaplásico aberrante que evolutivamente tiende a ser displásico.

Por todo ello, se necesitan estudios futuros probablemente moleculares para clarificar el comportamiento a largo plazo de la metaplasia intestinal de vejiga ${ }^{13}$.

\section{REFERENCIAS}

1. BULLOCK PS, THONI DE, MURPHY M.: The significance of colonic mucosa (intestinal metaplasia) involving the urinary tract. Cancer 1987; 59: 2086-2090.

2. SUSMANO D, RUBESNSTEIN A, DAKIN A, LLOYD F.: Cystitis glandularis and adenocarcinoma of the bladder. J Urol 1971; 105: 671-674.

3. BELL TE, WENDEL RG.: Cystitis glandularis: benign or malignant?. J Urol 1968; 100: 462-465.

4. CORICA FA, HUSMANN DA, CHURCHIL BM, YOUNG RH, PACELLI A, LÓPEZ-BELTRÁN A, BOSTWICK D.: Intestinal metaplasia is not a strong risk factor for bladder cancer: study of 53 cases with long-term follow-up. Urology 1997; 50: 427-431.

5. HEYNS CF, DE KOCK MLS, KIRSTEN PH, VAN VELDEN JJ.: Pelvic lipomatosis associated with cystitis glandularis and adenocarcinoma of the bladder. J Urol 1991; 145: 364-366.

6. VICENTE J, ALGABA F.: Cistopatía quística y glandular. En "Semiología diagnóstica endovesical. Valoración endoscópica y microscópica”. Barcelona. Pulso Ediciones 1999: 82-92.
7. YOUNG RH, BOSTWICK D.: Florid cystitis glandularis of intestinal type with mucin extravasation: a mimic of adenocarcinoma. Am J Surg Pathol 1996; 20: 1462-1468.

8. SHAW J, JISLASON G, IMBRIGLIA J.: Transition of cystitis glandularis to primary adenocarcinoma of the bladder. J Urol 1958; 79: 815-822.

9. HAMPSON SJ, FALZON M, COWIE AG.: Intestinal metaplasia of the bladder: implications for management. Br J Urol 1992; 69: 323-324.

10. EDWARS P, HURM R, JAESCHKE WH.: Conversion of cystitis glandularis to adenocarcinoma of the bladder. J Urol 1972; 108: 568-570.

11. THRASHER JB, PERZ LM, HUMPHREY PA, ANDERSON EE.: Cystitis glandularys: transition to adenocarcinoma of urinary bladder. $N$ Carolina Med $J$ 1994; 55: 562-564.

12. ADEGBOYEGA PA, ADEKUNLE A.: Tubulovillous adenoma of the urinary bladder. Mod Pathol 1999; 12: $735-738$.

13. PANTUCK AJ, BANCILA E, DAS KM, AMENTA PS, CUMMINGS KB, MARKS M, WEIS RE.: Adenocarcinoma of the urachus and bladder expresses a unique colonic epithelial epitope. An immunohistochemical study. J Urol 1997; 158: 17221727.

14. MURPHY DP, PANTUCK AJ, AMENTA PS, DAS KM, CUMMINGS KB, KEENEY GL, WEISS RE.: Female urethral adenocarcinoma: immunohistochemical evidence of more than 1 tissue of origin. J Urol 1999; 161: 1881-1884.

Dra. R. Guarch Troyas

Servicio de Anatomía Patológica

Hospital Virgen del Camino

C/ Irunlarrea, 4

31108 Pamplona (Navarra)

(Trabajo recibido el 16 julio de 2002) 